\title{
Analysis of Stability Criteria in the Host-Parasitoid System - A Mathematical Approach
}

\author{
Madhusudanan.V², Vijaya Lakshmi .G.M², Jayanthi .C.E ${ }^{3}$, Gunasekaran .M ${ }^{4}$,* \\ Department of Mathematics \\ ${ }^{1}$ S.A. Engineering College, Chennai-600 54 \\ ${ }^{2}$ Indira Institute of Engineering and Technology, Thiruvallur-631 203 \\ ${ }^{3}$ B.K.R. College of Education, Tiruttani-631 209 \\ ${ }^{4}$ Sri Subramaniya Swamy Government Arts College, Tiruttani-631 209
}

\begin{abstract}
Nicholson Bailey model (NB-1930) was developed to describe the population dynamics of host parasite system. Host parasite models are similar to host parasitoid model except that the parasite does not kill the host [1]. The NB model does not allow for stable host-parasitoid interactions due to potential fecundity of parasites is not limited [7]. In this paper the modified NB model is applied to host parasitoid system and the stability criteria were analysed and illustrated.
\end{abstract}

Keywords: Host-parasitoid system, Nicholson-Bailey model, Stability analysis.

\section{Introduction}

Population models or reaction-diffusion systems have attracted enormous interest both in the mathematical community and as abstract versions of real biological dynamics [8].Every year, millions of hectares of agricultural land are treated with released Trichogramma wasps [15]; for instance, to protect sugar cane from the sugar cane borer, Chilo spp., in China, or to protect corn fields from the European corn borer, OstrinianubilalisHübner, in Western Europe. Theoretical studies of host-parasitoid interactions go back to Nicholson and Bailey $[3,9,13]$. One of the earliest realistic models of two-species interactionwas developed by Nicholson and Bailey to describe a simple discrete-time model for the population dynamics of insect hosts and their parasitoid that has since become one of the classical models of population biology.Parasitoid [5], such as parasitic wasps, lay eggs into their hosts and thus the completion of the parasitoid life cycle requires that their hosts be killed. Parasitoid is seem like parasites as they grow inside a host, but also seems like predators in that they are obligate killers of their host. The discrete N-B model does not allow for stable host-parasitoid interactionswhich is an example for an unstable oscillation [4]. In this paper to analyse stability, the N-B model has been extensively modified to add new elements of host and parasitoid.In the present investigation, an existing modified Nicholson Bailey model (NB model) is examined for stability criteria in host-parasitoid system [7]. The stability analysis that we carried out analytically has also been presented with numerical simulations.

\section{The model}

We have the discrete generation, host-parasitoid Nicholson-Bailey model for two-dimensional system of difference equations

$$
\begin{aligned}
& H_{n+1}=b H_{n} e^{-a p_{n}} \\
& P_{n+1}=c H_{n}\left(1-e^{-a p_{n}}\right)
\end{aligned}
$$

Our analysis can be carried out most precisely by reference to an appropriate host-parasitoid system model. We shall use Modify Nicholson-Bailey model to obtain bounded dynamics where the parameters discussed earlier. Assume the host has bounded dynamics in absence of parasitoid. That is it has self-regulation(density dependence). For example, assume host dynamics are inherently logistic[10].Forexample, assume host dynamics are inherently logistic

Where

$$
\begin{aligned}
& H_{n+1}=b \frac{1}{1+d H_{n}} H_{n} e^{-a P_{n}} \\
& P_{n+1}=c H_{n}\left(1-e^{-a P_{n}}\right)
\end{aligned}
$$

\footnotetext{
$H_{n} \quad$-host population at time $\mathrm{n}$

$P_{n} \quad$-parasitoid population at time $\mathrm{n}$

$H_{n+1}$.host population size at time $\mathrm{n}+1$ in terms of the population at time $\mathrm{n}$.
} 
$P_{n+1} \quad$ parasitoid population size at time $\mathrm{n}+1$ in terms of the population at time $\mathrm{n}$.

b the fecundity of the host at time $n+1$.

c - average number of successful eggs laid per parasitoid.

$\mathrm{e}^{-a p_{n}} \quad$ - random encounter between hosts and parasitoids the probability (Poisson distribution) that a host escapes parasitism (ie.,) the probability of host not infected.

$\left(1-\mathrm{e}^{-a p_{n}}\right) \quad$ - probability of host to become infected.

a -proportionality constant.

\section{Fixed point and Local stability}

We now study the existence of fixed points of the system (2), particularly we are interested in the interior fixed point to begin and we list all possible fixed points.

i) $E_{0}=(0,0)$ is trivial fixed point

ii) $E_{1}=\left(H^{\bullet}, P^{\bullet}\right)$ is the interior fixed point where

$$
H^{\bullet}=\frac{\ln b}{a c\left(1-e^{-a P}\right)}
$$

And $P^{\bullet}=a^{-1} \ln b$

Theorem: If $p(\lambda)=\lambda^{3}+B \lambda^{2}+C \lambda+D$ be the roots of $p(\lambda)=0$ then the following statements are true

a) If every root of the equation has absolute value less than one, then the fixed point of the System is locally asymptotically stable and fixed point is called a sink.

b) If at least one of the roots of equation has absolute value greater than one then the fixed Point of the system is unstable and fixed point is called saddle.

c) If every root of the equation has absolute value greater than one then the system is a source.

d) The fixed point of the system is called hyperbolic if no root of the equation has absolute value equal to one if there exists a root of equation with absolute value equal to one then the fixed point is called non-hyperbolic.

\section{Dynamic behaviour of the model}

In this subsection we investigate the local behaviour of the model (1) and (2) around Each fixed point. The local stability analysis of the model (1) and (2) can be studied by computing the variation matrix corresponding to each fixed point . The variation matrix of the model at state variable is given by

$$
\begin{gathered}
H(t+1)=F_{1}\left(H_{1}, P_{1}\right) \\
P(t+1)=F_{2}\left(H_{2}, P_{2}\right)
\end{gathered}
$$

For which the Jacobian matrix is given by

$$
J(H, P)=\left(\begin{array}{ll}
\frac{\partial F_{1}}{\partial H} & \frac{\partial F_{1}}{\partial P} \\
\frac{\partial F_{2}}{\partial H} & \frac{\partial F_{2}}{\partial P}
\end{array}\right)
$$

Where

$$
\begin{aligned}
& \frac{\partial F_{1}}{\partial H}=\frac{b e^{-a P}}{(1+d H)^{2}} \\
& \frac{\partial F_{1}}{\partial P}=\frac{-a b H e^{-a P}}{(1+d H)} \\
& \frac{\partial F_{2}}{\partial H}=c\left(1-e^{-a P}\right) \\
& \frac{\partial F_{2}}{\partial P}=c H\left(a e^{-a P}\right)
\end{aligned}
$$


Proposition 1The fixed point $E_{0}$ is locally asymptotically stable if $b<1$, otherwise unstable $[11,12,14]$.

Proof:

In order to prove the result we estimate the eigenvalues of the jacobian matrix $\mathrm{J}$ at $E_{0}$ is given by $J\left(E_{0}\right)=\left(\begin{array}{ll}b & 0 \\ 0 & 0\end{array}\right)$

Hence the eigen values of $\mathrm{J}$ at $E_{0} \quad$ are $\lambda_{1}=b$ and $\lambda_{2}=0$

Thus it is stable node if $b<1$.If $b>1$ the fixed point $E_{0}$ is unstable.

Proposition 2 The fixed point $E_{1}$ stable [2] if satisfy the condition $\lambda_{1}<1, \lambda_{2}<1$ otherwise un stable if $\lambda_{1,}>1$.

Proof: In order to prove the result consider the matrix' $\quad A=\left(\begin{array}{ll}G_{11} & G_{12} \\ G_{21} & G_{22}\end{array}\right)$

$$
\begin{gathered}
\text { Where } G_{11}=\left.\frac{\partial F_{1}}{\partial H}\right|_{E_{1}}=b^{-1} \\
\begin{array}{c}
G_{12}=\left.\frac{\partial F_{1}}{\partial P}\right|_{E_{1}}=-a d^{-1}(b-1) \\
G_{21}=\left.\frac{\partial F_{2}}{\partial H}\right|_{E_{1}}=c \\
G_{22}=\left.\frac{\partial F_{2}}{\partial P}\right|_{E_{1}}=a c d^{-1}(b-1)
\end{array}
\end{gathered}
$$

The characteristic equation is $\quad|A-\lambda I|=0$

This may be rewritten in the form

$$
\Rightarrow\left(\begin{array}{cc}
G_{11}-\lambda & G_{12} \\
G_{21} & G_{22}-\lambda
\end{array}\right)=0
$$

$$
\lambda^{2}-B \lambda+C=0
$$

Where $\mathrm{B}=$ Trace of $\mathrm{A}=G_{11}+G_{22}$

$$
\begin{aligned}
= & \frac{d+a c b(b-1)}{b d} \\
\mathrm{C}=|\mathrm{A}|= & G_{11} \cdot G_{22}-G_{12} \cdot G_{21} \\
= & \frac{a c\left(b^{2}-1\right)}{b d}
\end{aligned}
$$

The roots of the equation (9) are

$$
\begin{gathered}
\lambda_{1}=a c d^{-1}(b-1) \\
\lambda_{2}=b^{-1} \\
\text { Now } \lambda_{1}<1 \text { for } a c d^{-1}(b-1)<1 \\
\Rightarrow b<1+a^{-1} c^{-1} d
\end{gathered}
$$

Therefore $\lambda_{1}<1$ for $b<1$ and $\lambda_{2}<1 \Rightarrow b^{-1}<1$ for $b>1$

Hence both equations are satisfied in which host-parasitoid equilibrium will be stable.Also if $\lambda_{1}>1$ then the host-parasitoid equilibrium will be unstable. 


\section{Numerical simulation}

In this section we give the numerical simulations to verify our theoretical results proved in the previous section by using MATLAB programming. We also confirm the results by visual representation of the system for some values of parameters.

The co-existence between host and parasitoid appears to be $|\lambda|<1$, for some values of parameters by taking $\mathrm{b}=0.5,1.54,1.75 ; \mathrm{H}=100, \mathrm{P}=10$ are stable which satisfies the condition in preposition (2) (Figs. 1, 2, 3, 4).
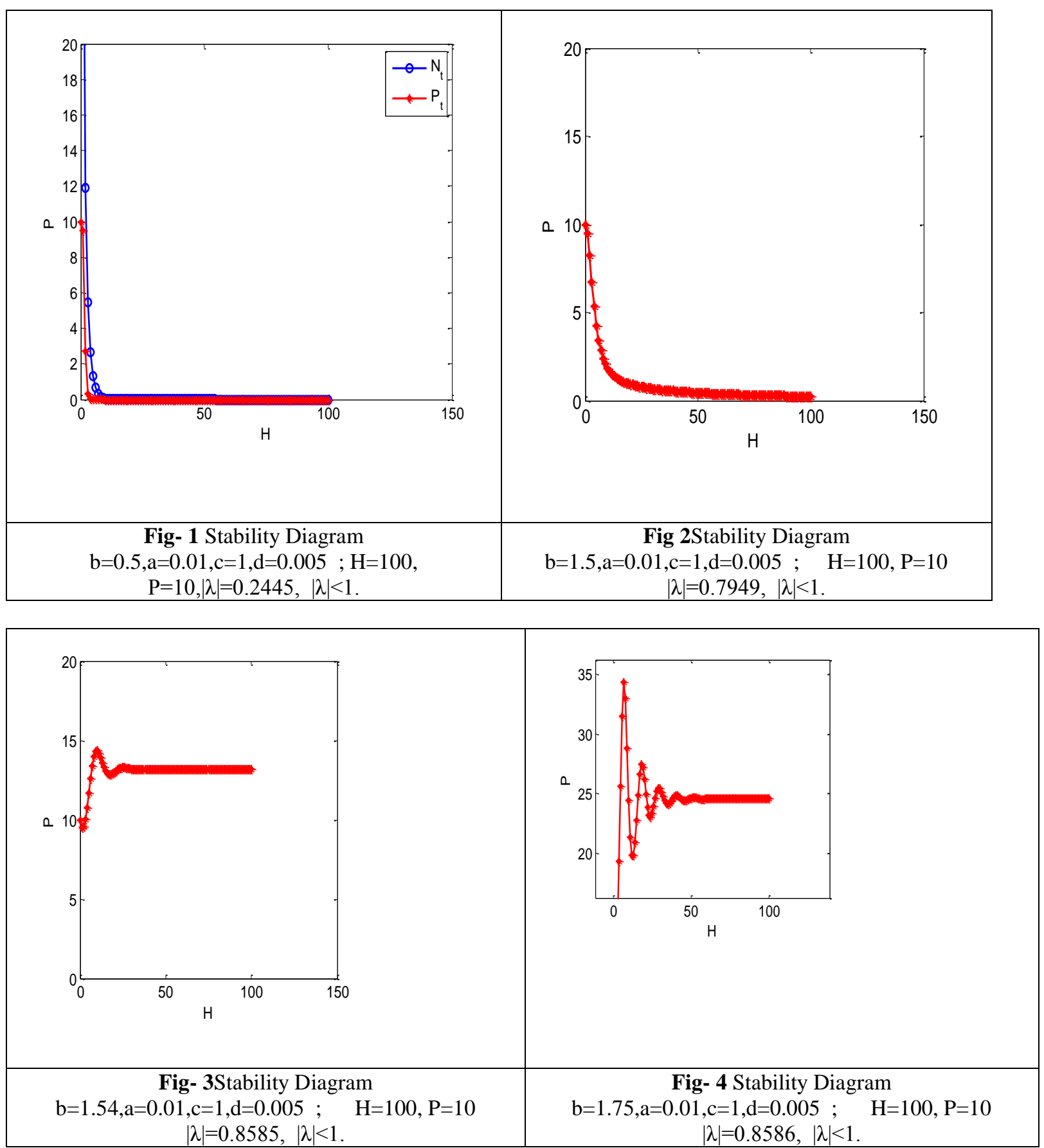

By taking the value $\mathrm{b}=3$ at $\mathrm{H}=100, \mathrm{P}=10$ we get $|\lambda|>1$, which shows the unstable equilibrium in the below Fig-5 but by increasing the encounter of host and parasite by $\mathrm{H}=140, \mathrm{P}=60$, we get the same condition as $|\lambda|>1$, unstable with a small change in $\lambda$ value, see Fig- 6 . 


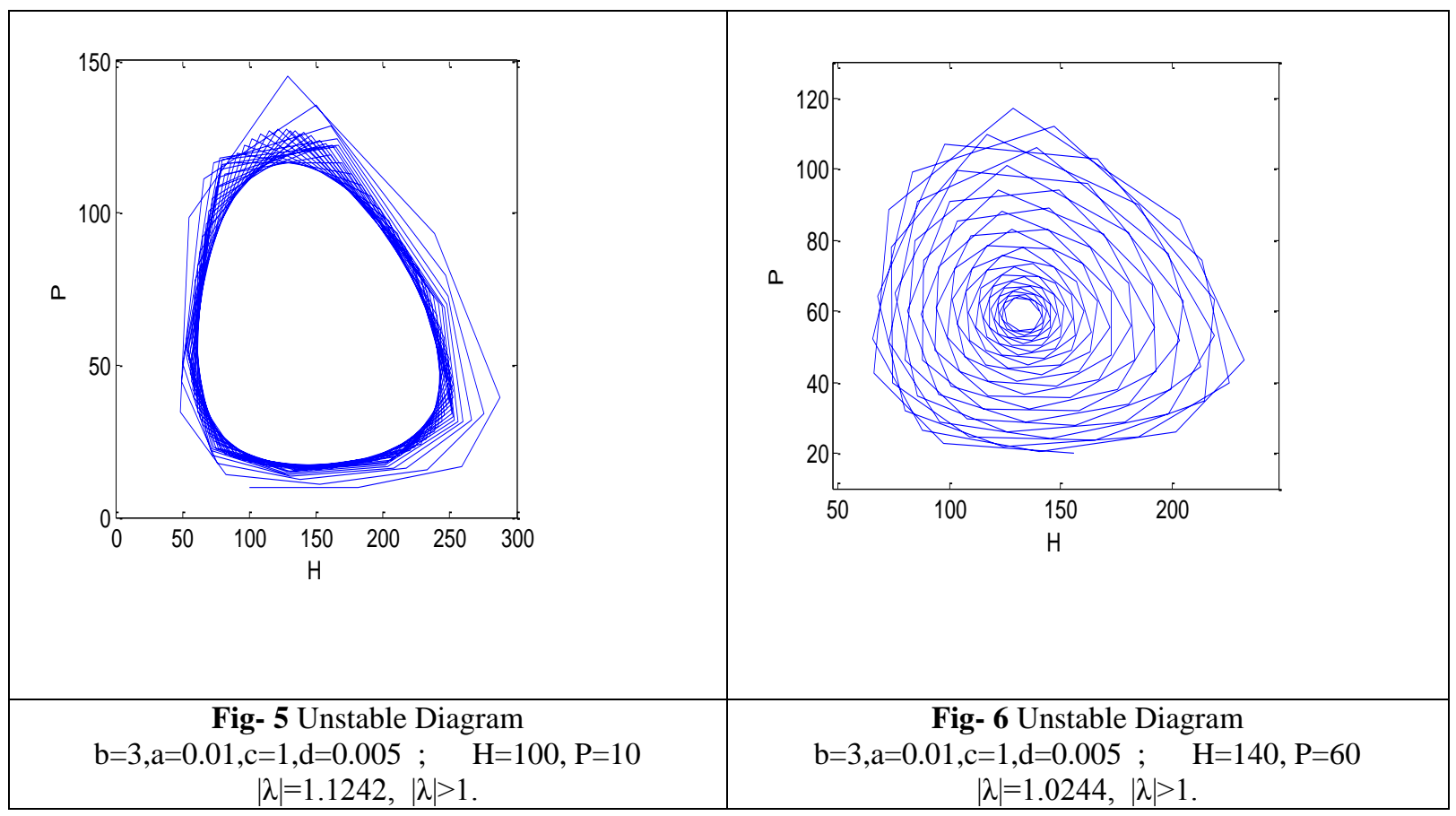

We conclude that the numerical simulations agree with the analytical results on modified N-B model.

\section{Conclusion}

This paper presents some innovative analysis with respect to previous studies on stability analysis in host parasitoid system [7,5,11,].The study of host-parasitoid with the help of modified N-B model yields interesting results. In general parasitoid lives in or on the host and necessarily kills the host. In the present study we discussed about the instability of host-parasitoid by using modified N-B model. We have introduced certain new parameters to modify N-B model and obtain equilibrium points. The numerical solution of the population size shows a succession of bifurcation. The effect of bifurcation in the model- I depends on the value of the intrinsic growth rate $\mathrm{b}$, which makes the system unstable or bifurcation. In modified N-B model the effect of intrinsic growth reduces the effect of bifurcation and results in the appearance of stable. It may thus be concluded that bifurcation of host offers a strong control in modified N-B model.

\section{References}

[1] Cheryl.J.Briggs and Martha.F.Hoopes: Stabilising effects in spatial parasitoid-host and predator - prey models: a review, 300, 299315 (2003).

[2] David Eberly: Stability analysis for system of Differential equations: 1-14, (2008).

[3] Elaydi.S: An introduction to difference equations. Springer, Berlin (2000)

[4] Hassell MP: Insecticides in host-parasitoid interactions. Theoretical population Ecology, 26(3), 378-386 (1984).

[5] Hassell. MP, comins. HN: Discrete time model for two-species competition, Theor. Population Biology 9, 202-221 (1976)

[6] J.D. Murray, Mathematical Biology, Vol. I, Springer, NewYork, 44-59, (1989).

[7] Jose D. Flores: Mathematical modelling, Nicholson Bailey model, (2011) 14-28.

[8] M.Gatto, G.A. De Leo: Interspecific competition among macro parasites in a density-dependent host population, 467-490 (1998)

[9] Mattipeltomaki, villevuorinen, MikkoAlara: Host-Parasite model on graph, 1, 1 (2013)

[10] May. R.M : Simple mathematical models with very complicated dynamics. 1, 1-16 (1976)

[11] May.R.M ,Oster.G.F: Bifurcations and dynamics complexity in simple ecological model 110, 573-599, (1976)

[12] Misra .JC, Mitra .A: Instabilities in single-species and host parasite systems: period- doubling bifurcations and chaos. Compt. Math. Appl.52, 525-53 (2006)

[13] Robert M. May: Host parasitoid systems in patchy environment: A phenomenological model 833-843(1978).

[14] SebastianBonhoeffer: Unstable oscillations and spatial structure: The Nicholson Bailey model, Level 2 module in "Modelling course in population and evolutionary biology" (2011) 1-16.

[15] Robert M. May: Host parasitoid systems in patchy environment: A phenomenological model 833-843(1978).

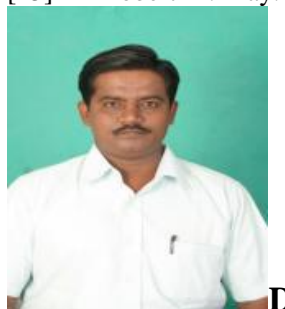

Dr M Gunasekaran received BSc, MSc and MPhil in Mathematics from Bharathidasan University, Tiruchirapalli,Tamilnadu in 1989, 1991 and 1992 rerspectively. He has completed his PhD degree in 
Mathematics-Zoology from university of Madras Chennai. He is a Professor of Mathematics with 22 years of teaching experience and now he is working in Sri Subramaniya Swamy Government Arts College, Tiruttani. His areas of research are Drug designing, Mathematical models in Biology and Queuing Models. He has published around 14 international journals, National and International Conferences. He has supervised 18 MPhil scholars in the area of various Queuing models. 